\title{
Anestesia y déficit de glucosa-6-fosfato deshidrogenasa. Revisión a propósito de un caso
}

Molero Díez YB, González Rodríguez JL.

Hospital Virgen de la Concha, Zamora.

\section{Resumen}

El déficit de glucosa-6-fosfato deshidrogenasa es el defecto enzimático más frecuente de los glóbulos rojos en los seres humanos. Esta alteración está relacionada con un defecto enzimático en la vía de las pentosasfosfato y cursa con hemólisis. Evitar posibles desencadenantes de las crisis hemolíticas es la mejor estrategia de prevención; para ello, el anestesiólogo tiene que conocer adecuadamente los fármacos empleados para el procedimiento anestésico y cuales son aptos para su empleo en estos pacientes. Se presenta en el artículo una revisión sobre este déficit a propósito de un caso de un paciente que acude a someterse a una intervención quirúrgica con anestesia general.

\section{Introducción}

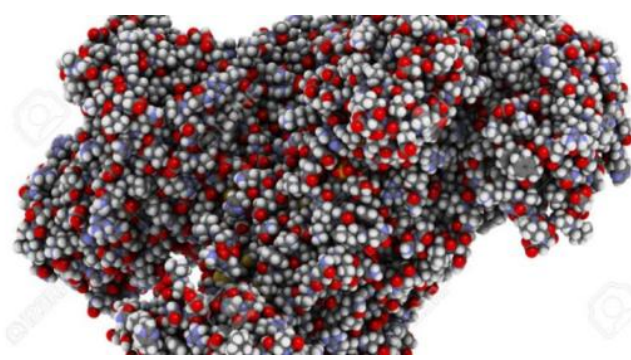

El déficit de glucosa-6-fosfato deshidrogenasa es el defecto enzimático más frecuente de los glóbulos rojos en los seres humanos. Esta alteración está relacionada con un defecto enzimático en la vía de las pentosas-fosfato y cursa con hemólisis. Evitar posibles desencadenantes de las crisis hemolíticas es la mejor estrategia de prevención; para ello, el anestesiólogo tiene que conocer adecuadamente los fármacos empleados para el procedimiento anestésico y cuales son aptos para su empleo en estos pacientes. Se presenta en el artículo una revisión sobre este déficit a propósito de un caso de un paciente que acude a someterse a una intervención quirúrgica con anestesia general.

\section{Caso clínico}

Paciente varón de 30 años, $75 \mathrm{~kg}$ de peso, de origen italiano, diagnosticado de DG6PDH mediante cribado neonatal, acude al Servicio de Cirugía remitido desde atención primaria por presentar una masa axilar izquierda junto con pérdida de $8 \mathrm{~kg}$ de peso en 6 meses. La tomografía computarizada reveló una tumoración axilar de $8 \times 8 \mathrm{~cm}$ adherida a planos, por lo que se decide su escisión quirúrgica. El paciente no había presentado ningún episodio hemolítico ni de ictericia previamente. Se intervino de una amigdalectomía y septorrinoplastia sin disponer de informes por no haberse realizado dichas intervenciones en nuestro centro (Hospital Virgen de la Concha, Zamora). 
Los valores analíticos preoperatorios estaban dentro de los límites normales (Hb: 13,8 g/dL, Hto: 42\%, plaquetas: $243 \times 10^{9} / \mathrm{L}$, TP:97\%, TTPA: $34 \mathrm{~s}$ )

La inducción anestésica se Mediante la administración de propofol con propofol a $2,5 \mathrm{mg} / \mathrm{kg}$ y fentanilo a 1 $\mu \mathrm{g} / \mathrm{kg}$. Se insertó una mascarilla laríngea Ambú Auragain ${ }^{\circledR} \# 4$ sin precisar de relajación neuromuscular.

Los parámetros ventilatorios que se fijaron fueron FiO2: 0,6 (mezcla de aire y oxígeno) volumen corriente: $8 \mathrm{ml} / \mathrm{kg} \mathrm{y}$ frecuencia respiratoria de 12 respiraciones por minuto.

El mantenimiento se estableció mediante técnica intravenosa total (TIVA) de propofol a $6 \mu \mathrm{g} / \mathrm{kg} / \mathrm{h}$ para el mantenimiento y bolos intermitentes de fentanilo de $1 \mu \mathrm{g} / \mathrm{kg}$ cada 30 minutos. El paciente permaneció estable hemodinámicamente en todo momento.

Se realizó una monitorización intraoperatoria estándar con electrocardiograma continuo (ECG), saturación de oxígeno ( $\mathrm{SpO} 2)$, presión arterial no invasiva, y end-tidal de $\mathrm{CO} 2$ (EtCO2). Se infundieron $650 \mathrm{ml}$ de suero salino fisiológico con calentador de fluidos. Se utilizó manta térmica durante toda la intervención. La pérdida hemática fue mínima. Tras los 80 minutos de intervención, $y$ administrándole 1 gr intravenoso de paracetamol previo a la educción, el paciente fue conducido a la unidad de recuperación postanestésica, donde tras 2 horas permaneció sin ninguna complicación, trasladándole a la planta de hospitalización de cirugía. Tras 3 días de estancia hospitalaria el paciente fue dado de alta a su domicilio sin ninguna clínica compatible con anemia hemolítica.

\section{Fisiopatología}

Para saber dónde actúa esta enzima, vamos a hacer un breve repaso fisiopatológico de la vía de las pentosasfosfato que se encargan del metabolismo oxidativo celular, ya que la enzima es clave en esta ruta metabólica (figura 1). ${ }^{(1,4)}$

La glucosa-6-fosfato deshidrogenasa (G6PDH) es la encargada de canalizar el paso oxidativo de glucosa-6-fosfato a 6fosfogluconato, además también interviene en la reducción de la nicotinamida adenina dinucleótido fosfato (NADP) hacia nicotinamida adenina dinucleótido fosfato reducida (NADPH), ya por último es un cofactor básico en el metabolismo del glutation, encargado de proteger frente a noxas endógenas $\mathrm{y}$ exógenas. $\mathrm{El}$ eritrocito contiene abundante glutatión reducido para defenderse contra estímulos oxidativos, si la enzima que permite el paso de glutatión oxidado hacia reducido, catalizada a su vez por la NADPH dependiente de G6PDH, se encuentra disminuida, no se va a realizar un correcto balance del estrés oxidativo del mismo precipitándose su hemólisis ${ }^{(5-}$ 7).

Al microscopio observaremos corpúsculos de Heinz junto con hematíes de aspecto "mordido"(3,8).

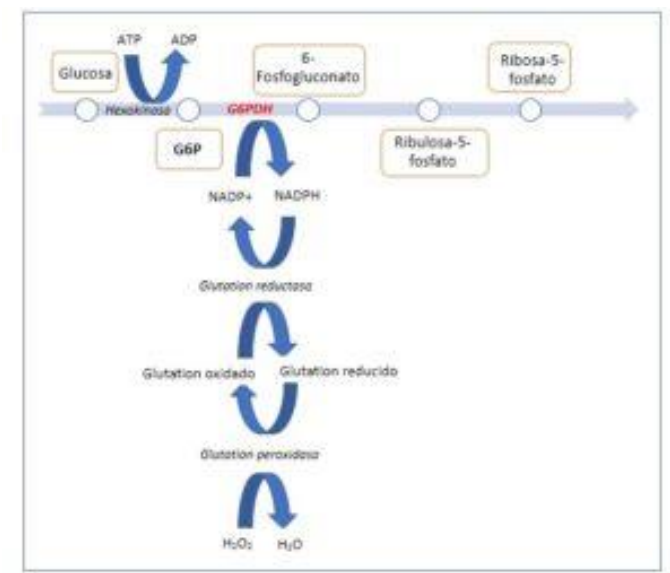

Figura 1: Ruta metabólica de las pentosas- 
fosfato. $\quad * \mathrm{G} 6 \mathrm{PDH}: \quad$ Glucosa-6-fosfato deshidrogenasa*

\section{Patogenia y aspectos genéticos}

El déficit de esta enzima, que está presente en todas las células del organismo en diferentes concentraciones, puede aumentar la vulnerabilidad de los hematíes ante determinadas sustancias oxidantes; si bien no conocemos el mecanismo exacto de como ocurre esta hemólisis, sí que sabemos que el resultado final es la desnaturalización de la hemoglobina y la reducción de la vida media de los glóbulos rojos $^{(1,7)}$.

El DG6PDH tiene un patrón de herencia recesivo ligado al cromosoma $\mathrm{X}$, el gen implicado es Xq28 ${ }^{(1,4)}$. El 90\% de los afectados son hombres (heterocigotos para el cromosoma $\mathrm{X}$ ), presentando clínica las mujeres solo cuando son homocigotas para dicho gen ${ }^{(3)}$.

Se trata de uno de los trastornos enzimáticos que presenta mayor variabilidad genética, habiéndose descrito múltiples mutaciones de dicha enzima, es por ello que han sido clasificadas en cinco grupos según el porcentaje de déficit y la severidad de la hemólisis que presentan por la Organización Mundial de la Salud (OMS) $^{(2)}$.

\section{Clínica}

Existen múltiples formas de presentación debido a la gran heterogeneidad genética que presenta este déficit ${ }^{(1)}$.

La mayor parte de los pacientes afectos por esta enfermedad suelen estar asintomáticos, pero también existen formas clínicas sintomáticas como son la anemia aguda hemolítica, el favismo, la anemia hemolítica congénita no esferocítica o la hiperbilirrubinemia neonatal, entre otras ${ }^{(6)}$.

La crisis hemolítica cursa con anemia, ictericia, orina colúrica y dolor abdominal $^{(5)}$. Las dos formas más frecuentes son la clase III de la OMS, también denominada G6PDH A-, que afecta fundamentalmente a personas de origen africano, la cual tiene efecto protector sobre el paludismo; y la clase II de la OMS, conocida a su vez como G6PDH A+ o forma mediterránea, que tiene mayor gravedad respecto a la hiperbilirrubinemia neonatal y mayor frecuencia de favismo ${ }^{(6)}$.

Evitar posibles desencadenantes de las crisis hemolíticas es la mejor estrategia de prevención en este tipo de pacientes. Si no está diagnosticada la enfermedad previamente, bien sea por cribado neonatal o por tener familiares afectos por dicha enfermedad, es posible que el paciente no sea consciente de su padecimiento hasta que no presenta la primera crisis.

Se deben evitar aquellos factores que provoquen un estrés oxidativo, entre los cuales se encuentran diversos fármacos. También se presentarán restricciones en cuanto a la alimentación, se deberá prescindir de habas o de productos que las contengan. Los procesos infecciosos a su vez podrán desencadenar una crisis en estos pacientes.

Algunos fármacos están perfectamente descritos como agentes oxidativos, sin embargo, otros, utilizados en nuestro campo de trabajo como pueden ser los anestésicos locales, no están tan estudiados $^{(2,9-12)}$ (Tabla 1). 


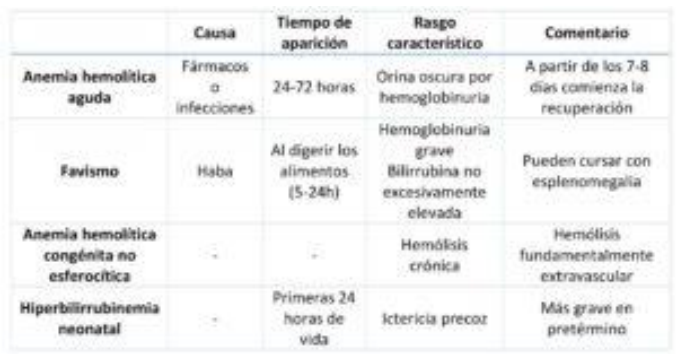

Tabla 1: Fármacos utilizados en anestesia y déficit de G6PDH

\section{Discusión}

El DG6PDH, a pesar de ser el defecto enzimático más frecuente en el ser humano, es una enfermedad poco frecuente en España, pero la cual no debemos olvidar debido a los flujos migratorios actuales ${ }^{(2)}$. En nuestro país su incidencia en adultos se estima entre un $0,1 \%-0,5 \%{ }^{(13)}$. El debut inicial suele ser tras una crisis hemolítica aguda en la que se estudia la funcionalidad de dicha enzima.

En España no se realiza un cribado neonatal de este déficit, en cambio este sí se realiza en el país natural de nuestro paciente, Italia. No hay un tratamiento específico, por lo que lo mejor para los enfermos es la prevención.

La infección es probablemente la causa más común de comienzo de una crisis hemolítica en este déficit ${ }^{(13)}$, sin embargo, dado nuestro campo de trabajo y sabiendo que algunos de los medicamentos empleados en anestesiología y en el control postoperatorio del dolor pueden causar hemólisis, debemos tener más en cuenta el efecto de todos los aquellos que empleamos en el periodo perioperatorio, siendo conscientes de utilizar aquellos que no provoquen un estrés oxidativo sobre las células.

A pesar de que ya se han realizado varios estudios con los agentes farmacológicos implicados en la anestesia, no son lo suficientemente concluyentes y siguen en constante actualización, debido a que algunos autores han relacionado de manera indirecta el G6PDH con la hipertermia maligna ${ }^{(14)}$.

Según la literatura consultada, el uso de propofol, fentanilo y paracetamol, empleando una mascarilla laríngea sin bloqueantes neuromusculares es una práctica segura en esta patología ${ }^{(9,11)}$.

El manejo perioperatorio de estos enfermos incluye reducir el estrés y el dolor que pudiera causar un estrés oxidativo, es por ello que consideramos que esta técnica propició un despertar tranquilo y sin dolor al joven, evitando así desencadenantes en el postoperatorio inmediato.

La anestesia general nos puede enmascarar los signos inmediatos de hemólisis mientras el enfermo está anestesiado, ya que la hipotensión que originaría puede ser achacada a otras causas. La aparición de hemoglobina en orina o libre en plasma pueden ser signos indirectos de una reacción hemolítica, ante estos indicios debemos suspender los fármacos que estemos administrando e intentar mantener el flujo urinario mediante la infusión de cristaloides y el uso de diuréticos ${ }^{(11)}$.

Por último, el uso de dexmedetomidina, agente anestésico con propiedades antioxidativas, induce una sedación que puede ser útil para evitar métodos anestésicos más invasivos en niños ${ }^{(15)}$, que requerirá más estudios para garantizar su seguridad en estas personas.

\section{Bibliografía}

1. Cappellini MD, Fiorelli G. Glucose-6phosphate dehydrogenase deficiency. 
The Lancet. 2008;371(9606):64-74. (PubMed) (HTML)

2. Group WW. Glucose-6-phosphate dehydrogenase deficiency. WHO Bulletin OMS. 1989;67:601-11. (PubMed) (HTML) (PDF)

3. Román Hernández C, Bonet de Luna C. Déficit de glucosa-6-fosfato deshidrogenasa: la peregrinación del chico con color. Pediatría Atención Primaria. 2016;18:349-54. (

4. Hsia YE, Miyakawa F, Baltazar J, Ching NS, Yuen J, Westwood B, et al. Frequency of glucose-6-phosphate dehydrogenase (G6PD) mutations in Chinese, Filipinos, and Laotians from Hawaii. Human genetics. 1993;92(5):470-6. (PubMed)

5. Frank JE. Diagnosis and management of G6PD deficiency. American family physician. 2005;72(7):1277-82. (PubMed) (HTML)

6. Bello Gutiérrez P, Mohamed Dafa L. Déficit de glucosa-6-fosfato deshidrogenasa: revisión a propósito de un caso. Pediatría Atención Primaria. 2015;17:361-8. (HTML)

7. Luzzatto L, Nannelli C, Notaro R. Glucose-6-Phosphate Dehydrogenase Deficiency. Hematology/Oncology Clinics. 2016;30(2):373-93. (PubMed)

8. Jollow DJ, McMillan DC. Oxidative stress, glucose-6-phosphate dehydrogenase and the red cell. Biological Reactive Intermediates VI: Springer; 2001. p. 595-605. (PubMed)

9. Glucose-6-phosphate dehydrogenase deficiency [Internet]. Orphananesthesia [cited 24/08/2018]. Available from: http://goo.gl/87vF0N.

10. Altikat S, Ciftci M, E Büyükokuroğlu M. In vitro effects of some anesthetic drugs on enzymatic activity of human red blood cell glucose 6-phosphate dehydrogenase2002. 67-71 p. (PubMed)

11. Elyassi CAR, Rowshan MHH. Perioperative management of the glucose-6-phosphate dehydrogenase deficient patient: a review of literature. Anesthesia progress. 2009;56(3):86-91. (PubMed) ( $\underline{\text { HTML) }}$ (PDF)

12. Youngster I, Arcavi L, Schechmaster R, Akayzen Y, Popliski H, Shimonov J, et al. Medications and Glucose-6Phosphate Dehydrogenase Deficiency. Drug Safety. 2010;33(9):713-26. (PubMed)

13. VERDUGO P, CALVANESE $M$, RODRÍGUEZ D, CÁRCAMO C. Deficiencia de glucosa 6 fosfato deshidrogenasa en niños: Caso clínico. Revista chilena de pediatría. 2014;85(1):74-9.

14. Basora M, Villalonga A, Ayuso M. Glucose-6-phosphate dehydrogenase deficiency: anesthetic implications. Revista espanola de anestesiologia y reanimacion. 1990;37(6):380.

15. Takahashi N, Ogawa T, Wajima Zi, Omi A. Dexmedetomidine-based intravenous anesthesia of a pediatric patient with glucose-6-phosphate dehydrogenase (G6PD) deficiency: A case report. Medicine. 2017;96(21):e6986. (PubMed) ( (PDF)

Correspondencia al autor

Yaiza Beatriz Molero Díez

yaiza923@hotmail.com

Residente de Anestesiología y Reanimación. Hospital Virgen de la Concha, Zamora.

José Luis González Rodríguez

jlgonzalezr@saludcastillayleon.es

LEA de Anestesiología y Reanimación.

Complejo Asistencial Universitario de

Salamanca.

Aceptado para el blog en diciembre de 2018. 